\title{
Estrutura trófica da ictiofauna em um reservatório do semiárido brasileiro
}

\author{
Jônnata F. Oliveira ${ }^{1,2}$, Antonio L. N. Moraes-Segundo², José L. C. Novaes' \\ Rodrigo S. Costa' , Jamillys S. França² \& Danielle Peretti
}

\author{
1. Laboratório de Ecologia de Peixes \& Pesca Continental, Departamento de Ciências Animais, Universidade Federal Rural do Semi-Árido, BR 110 Km 47, \\ Costa e Silva, 59625-900 Mossoró, RN, Brasil. \\ 2. Laboratório de Ictiologia, Departamento de Ciências Biológicas, Universidade do Estado do Rio Grande do Norte, Av. Prof. Antônio Campos, Costa e Silva, \\ 59625-620 Mossoró, RN, Brasil. (danielleperetti@uern.br)
}

Recebido 5 dezembro 2014

Aceito 23 abril 2016

DOI: $10.1590 / 1678-4766 e 2016001$

\begin{abstract}
Trophic structure of the ichthyofauna in a Brazilian semiarid reservoir. The establishment of the trophic structure of fish provides information on species autoecology and their role in the ecosystem, in addition to supporting conservation and management practices for sustainable use of native species. In the semiarid region of Rio Grande do Norte there are few studies of the natural diet of fish. In this context, this study aimed to characterize the trophic structure of the fish assemblage of the Santa Cruz reservoir, Apodi, state of Rio Grande do Norte, Brazil. Fish were collected quarterly (February, May, August and November) in eight points in the reservoir in 2011. After screening, biometric identification and analysis, stomach or intestinal contents were analyzed and food items identified were used for the calculation of the frequency of occurrence and volumetric whose association gives the Alimentary Index (IAi). The highest consumption of a particular item grouped the species into five trophic guilds, and the detritivore/iliophagous, insectivore and carcinophagous the most abundant in the months and collection points, compared to herbivorous and piscivorous. The high abundance of individuals in these guilds can be explained by opportunistic species and the capture facility and intake of resources, since detritus/sediment, insects and shrimp are abundant in the environment studied. Thus, this study provided information about the items that contribute to the diet species, making it possible to infer about the interactions, allowing in the future to understand the complexity of trophic relationships and to assist the management practices and conservation of environment.
\end{abstract}

KEYWORDS. Trophic guilds, fishes, Santa Cruz Reservoir.

RESUMO. O estabelecimento da estrutura trófica de peixes fornece informações sobre a autoecologia das espécies e seu papel no ecossistema, além de subsidiar práticas de conservação e manejo visando o uso sustentável das espécies nativas. Na região semiárida do Rio Grande do Norte existem poucos trabalhos sobre a alimentação natural de peixes. Neste contexto, o presente estudo visou caracterizar a estrutura trófica da assembleia de peixes do reservatório de Santa Cruz, Apodi, Estado do Rio Grande do Norte, Brasil. Os peixes foram coletados em oitos pontos, nos meses de fevereiro, maio, agosto e novembro de 2011. Após triagem, identificação e análise biométrica, os conteúdos estomacais ou intestinais foram analisados e, os itens alimentares identificados, utilizados para o cálculo das frequências de ocorrência e volumétrica cuja associação origina o Índice Alimentar (IAi). O maior consumo de um determinado item agrupou as espécies em cinco guildas tróficas, sendo os detritívoros/iliófagos, insetívoros e carcinófagos os mais abundantes entre os meses e pontos de coletas, em relação aos herbívoros e piscívoros. A elevada abundância de indivíduos nessas guildas pode ser explicada pelo caráter oportunista das espécies e, pela facilidade de captura e ingestão dos recursos, uma vez que detrito/sedimento, insetos e camarão são abundantes no ambiente estudado. Assim, o presente estudo forneceu informações sobre os itens que contribuem para as dietas das espécies, o que possibilitará inferir sobre as interações existentes, possibilitando no futuro entender a complexidade das relações tróficas e dar subsídios a práticas de manejo e conservação deste ambiente.

PALAVRAS-CHAVE. Guildas tróficas, peixes, Reservatório de Santa Cruz.

O estudo da alimentação natural de peixes e o estabelecimento da estrutura trófica fornecem informações relevantes sobre o funcionamento do ecossistema no qual estão inseridos, permitindo compreender a autoecologia das espécies e seu papel no ecossistema e identificar os fatores que determinam o padrão de alimentação do peixe ao longo de seu ciclo de vida. Esse conhecimento é indispensáveis para avaliar os processos que alteram o hábito alimentar das espécies frente a diferentes tipos de alterações ambientais, que promovem mudanças nas interações bióticas, sobretudo aquelas de natureza trófica (PINTo et al., 2011), o que pode levar indivíduos de uma mesma espécie a distinções espaciais 
e temporais relacionadas à alimentação.

WINDELL \& Bowen (1978) enfatizaram a importância na utilização desses estudos ao inseri-los como base para o entendimento das relações existentes entre a ictiofauna e seu hábitat, além de subsidiar práticas de conservação e manejo visando o uso sustentável das espécies nativas. No semiárido, especificamente no Rio Grande do Norte (RN), destacando-se estudos como os realizados na lagoa Boa Cica (CANAN et al., 1997), na lagoa Redonda (GURGEL et al., 1994, 1998), na lagoa do Jiqui (GURGEL \& CANAN, 1999), na lagoa de Extremoz (RAPOSO \& GURGEL, 2003), na lagoa do Piató (Gavilan-Leandro et al., 2009; Silva et al., 2012), no reservatório Riacho da Cruz (TeixeIRA \& Gurgel, 2004), no açude Corredor (Gurgel et al., 2002), no açude Marechal Dutra (Montenegro et al., 2011; PessoA et al., 2013), no rio Piranhas-Açu (MagalHães et al., 1990; GURGEL et al., 2002), no rio Ceará Mirim (ANDRADE et al., 2000; GuRgel et al., 2005; Dias \& Fialho, 2009) e no rio Seridó (Mendes et al., 2011).

Com base no levantamento bibliográfico realizado, não foi registrado trabalhos sobre a estrutura trófica de peixes no reservatório de Santa Cruz, em Apodi, RN. Este reservatório destaca-se no Estado pelas diversas atividades que ali se desenvolvem como: lazer, uma vez que a região a jusante é utilizada como balneário; abastecimento, contando com a construção de uma adutora, atividades de pesca artesanal realizadas por pescadores tradicionais e piscicultura, com tanques redes para o cultivo da tilápia, Oreochromis niloticus (Linnaeus, 1758), apoiados pelo Departamento Nacional de Obras Contras as Secas (DNOCS) e Serviço de Apoio às Micro e Pequenas Empresas (SEBRAE/RN).

Além de ser um ambiente modificado pelo barramento do rio, o que pode ter refletido em mudanças na composição e abundância de peixes constituintes do seu rio formador, é importante salientar que assim como alguns dos reservatórios do semiárido, o reservatório de Santa Cruz também receberá as águas do rio São Francisco (SANTANA FilHo, 2007). Além de modificar as características físico-químicas do ambiente, a transposição pode provocar situações indesejáveis na qualidade ou condições ambientais (AGRA FILHO, 2010), como na dinâmica populacional aquática, em especial na ictiofauna local devido à introdução de novas espécies.

As espécies introduzidas são um dos maiores problemas ambientais atuais globais (FERNANDES \& BARBOSA, 2012). Seja de forma acidental ou deliberada, a introdução apresenta efeitos indesejáveis sobre a biodiversidade, desde o nível genético até o de paisagens, podendo se tornar invasões biológicas e ocasionar mudanças que só serão percebidas em longo prazo e larga escala espacial, como a homogeneização biótica (Vitule \& Prodocimo, 2012), devido à redução ou extinção local de espécies (Espíndola et al., 2003), ocasionado pela competição por recursos, predação, inibição da reprodução e alteração do ambiente (AGOSTiNHO et al., 2007).

Portanto, como forma de contribuir com informações a respeito da biologia dos peixes nessa região, objetivase investigar a alimentação das espécies componentes da assembleia de peixes do reservatório de Santa Cruz, Rio Grande do Norte, a qual contribuirá para identificar as guildas tróficas e analisar as variações temporais e espaciais na abundância de indivíduos componentes das guildas.

\section{MATERIAL E MÉTODOS}

A região da bacia hidrográfica do rio Apodi-Mossoró, RN tem precipitação média anual de $750 \mathrm{~mm}$, concentrandose entre os meses de fevereiro e junho. O principal rio dessa bacia é o rio Apodi-Mossoró, com área de $4.246 \mathrm{~km}^{2}$, o qual apresenta 22 reservatórios (Almeida et al., 2006). Dentre esses empreendimentos, o reservatório de Santa Cruz, localizado no município de Apodi ( $05^{\circ} 39^{\prime} 01^{\prime \prime} \mathrm{S}, 37^{\circ} 47^{\prime} 56^{\prime \prime} \mathrm{O}$; Fig. 1) é o segundo maior do estado e o maior nesta bacia, com área de $3.413 \mathrm{~km}^{2}$, altura de $57,50 \mathrm{~m}$, capacidade máxima de 600 milhões de $\mathrm{m}^{3}$ e volume morto de 15 milhões de $\mathrm{m}^{3}$ de água (SEMARH, 2009). Teve sua construção iniciada em 1999 e finalizada em 2002, com a finalidade de fornecer água para irrigar 9.236 hectares na Chapada do Apodi, controlar as cheias, regular a vazão do Rio Apodi-Mossoró e garantir o abastecimento de água para 27 cidades do alto oeste potiguar (PACheco \& Baumann, 2006), constituindo-se como um dos reservatórios mais importantes para a região.

Em laboratório foram obtidos os dados de precipitação, fornecidos mensalmente pelo Instituto Nacional de Meteorologia - INMET $3^{\circ}$ Distrito de Meteorologia/ SEPRE, o que permitiu determinar os meses de fevereiro e maio como período chuvoso e os meses de agosto e novembro como seco (Tab. I).

Os peixes foram capturados em oito pontos distribuídos ao longo do reservatório (pontos 1 e 2, barragem; 3 e 4, parte central; 5 e 6, área de transição; 7 e 8, desembocadura do rio; Fig. 1), nos meses de fevereiro, maio, agosto e novembro de 2011. Em cada ponto, a coleta foi realizada com 11 redes de espera com malhas entre 12 a $70 \mathrm{~mm}$ entre nós adjacentes, com $15 \mathrm{~m}$ de comprimento e altura entre 1,8 a $2,0 \mathrm{~m}$ cada, totalizando $301,8 \mathrm{~m}^{2}$ de área de rede, instaladas às $17 \mathrm{~h}$ com revistas às $21 \mathrm{~h}$ e às $05 \mathrm{~h}$, permanecendo no ambiente por um período de $12 \mathrm{~h}$.

Os exemplares capturados foram transportados para o laboratório para identificação através de bibliografia (Rosa et al., 2003), sendo alguns exemplares enviados para confirmação por taxonomista da Universidade Federal da Paraíba (UFPB), onde os exemplares estão depositados na coleção ictiológica (número de catálogo: UFPB 8953 - 8997). Foram obtidos o comprimento total $(\mathrm{cm})$ e o peso total $(\mathrm{g})$ e em seguida os exemplares foram dissecados, sendo retirados o intestino de Loricariidae e os estômagos para as demais famílias. Os estômagos e intestinos foram fixados em formol a $10 \%$ durante $72 \mathrm{~h}$ e conservadas em álcool $70 \%$.

As análises do conteúdo estomacal ou intestinal foram realizadas sob estereomicroscópio e microscópio óptico e os recursos alimentares identificados, quando possível, até o menor nível taxonômico, utilizando-se MCCAFFERTY (1981), Higuti \& Franco (2001) e Merritt \& Cummins (1996) para invertebrados; BICUdo \& MENEzes (2006) para 


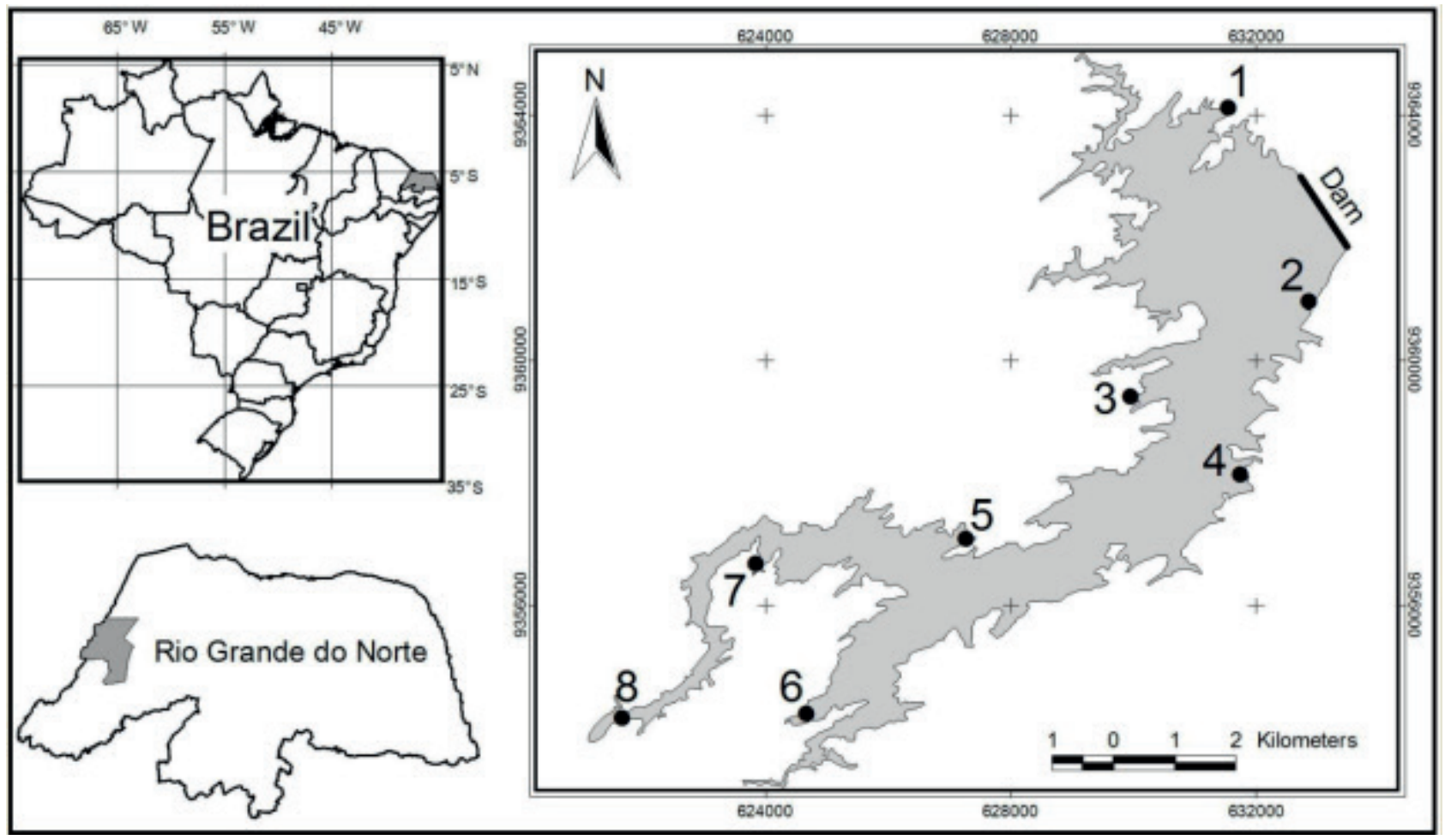

Fig. 1. Localização da área de estudo e dos pontos de coleta, reservatório de Santa Cruz, Rio Grande do Norte, Brasil.

Tab. I. Índice pluviométrico, volume de água acumulado no reservatório e percentual (\%) do volume de água acumulado nos meses de coleta (fevereiro, maio, agosto e novembro de 2011), no reservatório de Santa Cruz, Rio Grande do Norte, Brasil.

\begin{tabular}{lcccc}
\hline & Fevereiro & Maio & Agosto & Novembro \\
\hline Precipitação $(\mathrm{mm})$ & 307,6 & 212,4 & 21,3 & 0,0 \\
Volume Acumulado $\left(\mathrm{m}^{3}\right)$ & 476.203 .900 & 501.116 .440 & 489.651 .840 & 470.469 .600 \\
\% do volume acumulado & 79,00 & 83,53 & 81,65 & 78,45 \\
\hline
\end{tabular}

fitoplâncton e NEEDHAM \& NEEDHAM (1982) para organismos aquáticos em geral. Depois de identificados, o volume dos itens foi obtido de acordo com o tipo e dimensão do alimento por meio de provetas graduadas cujo volume é dado pelo deslocamento de líquido ou pelo método volumétrico rápido de Hellawell \& Abel (1971). Neste método, os itens alimentares são separados de acordo com o nível taxonômico e espalhados sobre uma placa de Petri milimetrada com alturas padronizadas em $1 \mathrm{~mm}^{3}$ e compactados contra lâminas de vidro de $1 \mathrm{~mm}$ de espessura, pelas laterais e por cima, formando blocos de $1 \mathrm{~mm}^{3}$; assim, os blocos formados tem seu volume mensurado em mL (BASTos et al., 2013).

Para os Loricariidae a análise foi feita a partir do conteúdo da porção anterior do lúmen, correspondendo a $10 \%$ do comprimento total do intestino (PERETTI \& ANDRIAN, 2004). Para Curimatidae, Prochilodontidae e Loricariidae obteve-se o volume total do conteúdo alimentar, o qual foi suspenso em álcool $70 \%$ e, parte da solução retirada foi investigada em lâmina sob microscópico. Para cada indivíduo, verificaram-se três lâminas e seus itens, depois de identificados, foram estimados visualmente em porcentagem. Os percentuais, associados ao volume total, correspondem ao volume do item (PERETTI \& ANDRIAN, 2004).

Dos dados da dieta foram calculadas as frequências de ocorrência e volumétrica cuja associação dá origem ao cálculo de Índice Alimentar (IAi) (KAWAKAMI \& VAZZOLER, 1980; ZaVAlA-CAMIN, 1996), em porcentagem: $I A i=[F o$. $F v / \Sigma(F O . F v)] \times 100$, onde: $I A i=$ Índice Alimentar; $F O$ $=$ frequência de ocorrência; $F v=$ frequência volumétrica.

Para avaliar a similaridade alimentar entre as espécies e agrupá-las nas guildas tróficas, foram utilizados os valores de IAi de cada espécie (apenas para espécies que possuíam mais de quatro estômagos com graus de repleção 1, 2 ou 3 ) em uma matriz de similaridade utilizando o índice de MorisitaHorn, através da análise de agrupamento não hierárquica com base no método de ligação UPGMA. Comparou-se a matriz de similaridade com uma matriz cofenética para testar possíveis distorções no processo de construção do dendrograma (o valor próximo à unidade indica melhor representação) (CRUZ \& CARNEIRO, 2003; Ximenes et al., 2011). Para avaliar se houve diferenças significativas na alimentação entre as guildas identificadas pela análise de agrupamento, foi realizada uma análise de similaridade (ANOSIM) com aplicação do índice de similaridade de Bray-Curtis.

Para verificar se ocorre variação temporal e espacial na abundância de indivíduos que compõem as guildas tróficas, 
os dados foram ordenados em análise de escalonamento multidimensional não métrico (NMDS) com base no índice de Bray-Curtis, usando a abundância de indivíduos por guilda trófica entre os meses e pontos de coleta. Com o objetivo de avaliar diferenças na abundância de indivíduos entre as guildas foi realizada a análise de variância multivariada permutacional (PERMANOVA), utilizando o índice de similaridade de Bray-Curtis. Para as análises dos dados foi utilizado o programa PAST versão 2.14 (HAMMER et al., 2001).

\section{RESULTADOS}

Foram coletados 2.526 exemplares distribuídos em três ordens, nove famílias e 19 espécies. Somente dez espécies apresentaram mais de quatro estômagos com conteúdo alimentar, sendo, portanto, realizada a análise de similaridade e categorização trófica apenas para estes indivíduos. As espécies mais abundantes foram Curimatella lepidura, Hypostomus pusarum e Triportheus signatus, respectivamente e, em menor número Astyanax bimaculatus e Loricariichthys platymetopon (Tab. II).

A dieta dos peixes apresentou 11 recursos com diferentes origens - animal, vegetal, sedimentos e detritos (Tab. III). Os itens de origem animal foram: (a) insetos, com a maior diversidade e em diferentes estágios de desenvolvimento:- Odonata (adultos e ninfas), Coleoptera, Hemiptera e Orthoptera (adultos), Trichoptera (pupa e adulto), Ephemeroptera (ninfa) e Diptera (larvas de Chironomidae e Chaoboridae); (b) anelídeos (cerdas de Oligochaeta); (c) peixes (musculatura e espinhas); (d) camarão (Machrobacium amazonicum Heller, 1862); (e) moluscos (Gastropoda, Melanoides tuberculata Muller, 1774); (f) outros invertebrados: restos de insetos (asas, pernas e partes de exoesqueleto cujas ordens não foram passíveis de identificação), (g) ovos de artrópodes e partes de aracnídeos (Araneae); (h) zooplâncton - microcrustáceos (Conchostraca,

Tab. II. Peixes estudados no reservatório de Santa Cruz, Rio Grande do Norte, Brasil (N, número de indivíduos coletados; N\%, porcentagem de indivíduos coletados; E, número de estômagos analisados; E\%, porcentagem de estômagos analisados).

\begin{tabular}{|c|c|c|c|}
\hline Grupo taxonômico & $\mathrm{N}(\mathrm{N} \%)$ & $\mathrm{E}(\mathrm{E} \%)$ & Material examinado \\
\hline \multicolumn{4}{|l|}{ CHARACIFORMES } \\
\hline \multicolumn{4}{|l|}{ CURIMATIDAE } \\
\hline Curimatella lepidura (Eigenmann \& Eigenmann, 1889) & $945(39,8)$ & $42(10,8)$ & $\begin{array}{l}\text { BRASIL, Rio Grande do Norte: Santa Cruz, Apodi } \\
\text { (Barragem de Santa Cruz, } 05^{\circ} 45^{\prime} \text { S, } 37^{\circ} 49^{\prime} \mathrm{W} \text { ), } \\
\text { 05.II.2010, J. L. Novaes e R. Costa col. (UFPB 8983) }\end{array}$ \\
\hline \multicolumn{4}{|l|}{ CHARACIDAE } \\
\hline Astyanax bimaculatus (Linnaeus, 1758) & $12(0,5)$ & $4(1,0)$ & $\begin{array}{l}\text { BRASIL, Rio Grande do Norte: Santa Cruz, Apodi } \\
\text { (Barragem de Santa Cruz, } 05^{\circ} 48^{\prime} \text { 'S } 37^{\circ} 48^{\prime} \text { W), } \\
\text { 21.XI.2010, J. L. Novaes e R. Costa col. (UFPB 8965) }\end{array}$ \\
\hline \multicolumn{4}{|l|}{ TRIPORTHEIDAE } \\
\hline Triportheus signatus (Garman, 1890) & $326(13,7)$ & $45(11,6)$ & $\begin{array}{l}\text { BRASIL, Rio Grande do Norte: Santa Cruz, Apodi } \\
\left.\text { (Barragem de Santa Cruz, } 05^{\circ} 49^{\prime} \text { S } 37^{\circ} 52^{\prime} W\right) \text {, } \\
\text { 24.II.2011, J. L. Novaes e R. Costa col. (UFPB 8982) }\end{array}$ \\
\hline \multicolumn{4}{|l|}{ ANOSTOMIDAE } \\
\hline Leporinus piau (Fowler, 1941) & $97(4,0)$ & $33(8,5)$ & $\begin{array}{l}\text { BRASIL, Rio Grande do Norte: Santa Cruz, Apodi } \\
\left.\text { (Barragem de Santa Cruz, } 05^{\circ} 47^{\prime} \text { S } 37^{\circ} 49^{\prime} \mathrm{W}\right) \text {, } \\
\text { 06.II.2010, J. L. Novaes e R. Costa col. (UFPB 8987) }\end{array}$ \\
\hline \multicolumn{4}{|l|}{ ERYTRINIDAE } \\
\hline Hoplias gr. malabaricus (Bloch, 1794) & $64(2,7)$ & $22(5,7)$ & $\begin{array}{l}\text { BRASIL, Rio Grande do Norte: Santa Cruz, Apodi } \\
\text { (Barragem de Santa Cruz, } 05^{\circ} 48^{\prime} \text { S } 37^{\circ} 48^{\prime} \mathrm{W} \text { ), } \\
\text { 27.II.2011, J. L. Novaes e R. Costa col. (UFPB 8996) }\end{array}$ \\
\hline \multicolumn{4}{|l|}{ SILURIFORMES } \\
\hline \multicolumn{4}{|l|}{ LORICARIIDAE } \\
\hline Loricariichthys platymetopon Isbrücker \& Nijssen, 1979 & $18(0,8)$ & $13(3,4)$ & $\begin{array}{l}\text { BRASIL, Rio Grande do Norte: Santa Cruz, Apodi } \\
\text { (Barragem de Santa Cruz, 0545'S 3747’W), } \\
\text { 29.XI.2009, J. L. Novaes e R. Costa col. (UFPB 8993) } \\
\text { BRASIL, Rio Grande do Norte: Santa Cruz, Apodi }\end{array}$ \\
\hline Hypostomus pusarum (Starks, 1913) & $548(23,1)$ & $54(13,9)$ & $\begin{array}{l}\text { (Barragem de Santa Cruz, } 05^{\circ} 50^{\prime} \text { S } 37^{\circ} 54^{\prime} \text { 'W), } \\
\text { 24.II.2011, J. L. Novaes e R. Costa col. (UFPB 8984) }\end{array}$ \\
\hline \multicolumn{4}{|l|}{ AUCHENIPTERIDAE } \\
\hline Trachelyopterus galeatus (Linnaeus, 1766). & $77(3,2)$ & $30(7,7)$ & $\begin{array}{l}\text { BRASIL, Rio Grande do Norte: Santa Cruz, Apodi } \\
\text { (Barragem de Santa Cruz, 0545'S 3747’W), } \\
\text { 29.XI.2009, J. L. Novaes e R. Costa col. (UFPB 8961) }\end{array}$ \\
\hline \multicolumn{4}{|l|}{ PERCIFORMES } \\
\hline \multicolumn{4}{|l|}{ CICHLIDAE } \\
\hline $\begin{array}{l}\text { Cichla monoculus } \\
\text { (Spix \& Agassiz, 1831) }\end{array}$ & $44(1,8)$ & $10(2,6)$ & $\begin{array}{l}\text { BRASIL, Rio Grande do Norte: Santa Cruz, Apodi } \\
\text { (Barragem de Santa Cruz, } 05^{\circ} 50^{\prime} \text { S } 37^{\circ} 52^{\prime} \text { W), } \\
\text { 24.II.2011, J. L. Novaes e R. Costa col. (UFPB 8976) }\end{array}$ \\
\hline \multicolumn{4}{|l|}{ SCIAENIDAE } \\
\hline Plagioscion squamosissimus (Heckel, 1840) & $243(10,2)$ & $134(34,6)$ & $\begin{array}{l}\text { BRASIL, Rio Grande do Norte: Santa Cruz, Apodi } \\
\text { (Barragem de Santa Cruz, 0550’S } 37^{\circ} 54^{\prime \prime W), ~} \\
\text { 24.II.2011, J. L. Novaes e R. Costa col. (UFPB 8978) }\end{array}$ \\
\hline Total & $2374(100,0)$ & $387(100,0)$ & \\
\hline
\end{tabular}


Tab. III. Índice Alimentar (IAi) das dez espécies de peixes estudadas no reservatório de Santa Cruz, Rio Grande do Norte, Brasil (Ins, inseto; Ane, anelídeo; Mol, molusco; Cam, camarão; O.I., outros invertebrados; Pei, Peixe; Zoo, zooplâncton; Veg, vegetal; Fit, fitoplâncton; D.S., detrito/sedimento; M.D., material digerido). Em destaque: valores acima de $25 \%$.

\begin{tabular}{|c|c|c|c|c|c|c|c|c|c|c|c|}
\hline Espécie & Ins & Ane & Mol & Cam & O.I. & Pei & Zoo & Veg & Fit & D.S. & M.D. \\
\hline T. signatus & 56,0 & & & & 6,86 & 0,7 & 6,2 & 6,8 & & & 23,4 \\
\hline T. galeatus & 52,6 & & & 19,6 & 3,34 & 13,4 & & & & 6,8 & 4,2 \\
\hline P. squamosissimus & 2,1 & & & 97,2 & & 0,6 & & 0,02 & & 0,03 & \\
\hline L. piau & 0,01 & & 31,8 & 1,1 & & 26,7 & 0,8 & 39,1 & & & 0,4 \\
\hline A. bimaculatus & 15,6 & & & & & & & 84,4 & & & \\
\hline H. malabaricus & & & & 1,3 & 0,17 & 98,3 & & 0,1 & & & 0,1 \\
\hline C. monoculus & 0,1 & & 0,1 & 19,5 & & 80,0 & & 0,2 & & & \\
\hline H. pusarum & & & & & & & 0,1 & 3,4 & 20,52 & 76,0 & \\
\hline L. platymetopon & 0,1 & 5,1 & & & & & 0,7 & 5,0 & 9,47 & 79,5 & \\
\hline C. lepidura & & 0,1 & & & & & 3,9 & & 41,3 & 54,7 & \\
\hline
\end{tabular}

Copepoda e Cladocera), rotíferos e protozoários (tecamebas). Para os itens de origem vegetal foram registrados restos de folhas, frutos, sementes e fitoplâncton: Chlorophyceae filamentosas e esféricas (Pediastrum e Chlorella), além dos gêneros Echinospharella, Desmodesmus e Scenedesmus, Bacillariophyta (Cyclotella, Encyonema, Acanthocera, Fragilariforma, Eunotia, Achananthidium, Acantes, Melosira, Navicula, Aulacoseira, Cymbopleura, Tryblionella, Rhoicosphenia e Craticula), Zygnemaphyceae (Croasdalea) e Cyanophyceae (Coelomoron). Outras algas que apresentaram baixa ocorrência foram Euglenas, Chlamydophyceas, Dinophyceas e Rhodophyceas.

Foi descrito ainda o item detrito/sedimento, que diferem quanto sua proporção de material orgânico e inorgânico: o primeiro constituído predominantemente por material animal e vegetal em decomposição e o segundo com predomínio de material inorgânico (areia ou silte). Aqueles itens cuja identificação não foi possível devido ao alto grau de digestão foram classificados como material digerido.

A partir da análise de similaridade alimentar, as espécies foram classificadas em cinco guildas tróficas (Fig. 2): Detritívora/Iliófaga (C. lepidura, L. platymetopon e $H$. pusarum), Herbívora (L. piau e A. bimaculatus), Piscívora (Hypostomus pusarum e C. monoculus), Carcinófaga ( $P$. squamosissimus) e Insetívora (T. signatus e T. galeatus), sendo os grupos formados dissimilares em relação à composição dos recursos consumidos (ANOSIM R $=1 ; p=0,0002$ ).

A ordenação evidenciou predomínio das guildas detritívora/iliófaga, insetívora e carcinófaga (Fig. 3), apresentando diferenças na abundância de indivíduos entre as guildas (PERMANOVA $\mathrm{F}=5,73 ; p=0,0001)$. Em relação à variação espacial, a ordenação também evidenciou predomínio das guildas detritívora/iliófaga, insetívora e carcinófaga entre os pontos de coletas (Fig. 4), ocorrendo diferença na abundância de indivíduos que compõe as guildas nos locais amostrados (PERMANOVA F $=3,34 ; p=0,0003$ ).

\section{DISCUSSÃO}

As guildas tróficas de peixes em reservatórios tropicais são muito complexas; no entanto, os recursos alimentares são poucos numerosos, onde os principais itens encontrados na dieta constituem-se de vegetais, insetos aquáticos e terrestres e, detritos (recursos alóctones), além do plâncton, bentos e peixes (recursos autóctone) (GURGEL et al., 2005; HAHN \& Fugi, 2009). Resultado similar foi encontrado no reservatório de Santa Cruz (RN), sendo que o consumo mais elevado de determinado item agrupou as dez espécies nas guildas detritívora/iliófaga, insetívora, carcinófaga, onívora e piscívora.

As demais espécies não foram categorizadas devido à elevada frequência de estômagos vazios, que pode ocorrer devido ao tempo que os exemplares permanecem nas redes, pois o longo período de exposição pode estressar o animal capturado e, consequentemente, induzir a regurgitação e a expulsão do alimento (P. M. Stefani, observ. pess.). Além disso, a grande incidência de estômagos vazios pode também ser uma característica de espécies carnívoras (GERKING, 1994), pois o fato de itens alimentares ingeridos serem relativamente grandes, de alto valor nutricional e de fácil digestão, diminui o tempo dispendido para saciar um peixe com essa dieta (HAHN et al., 1999).

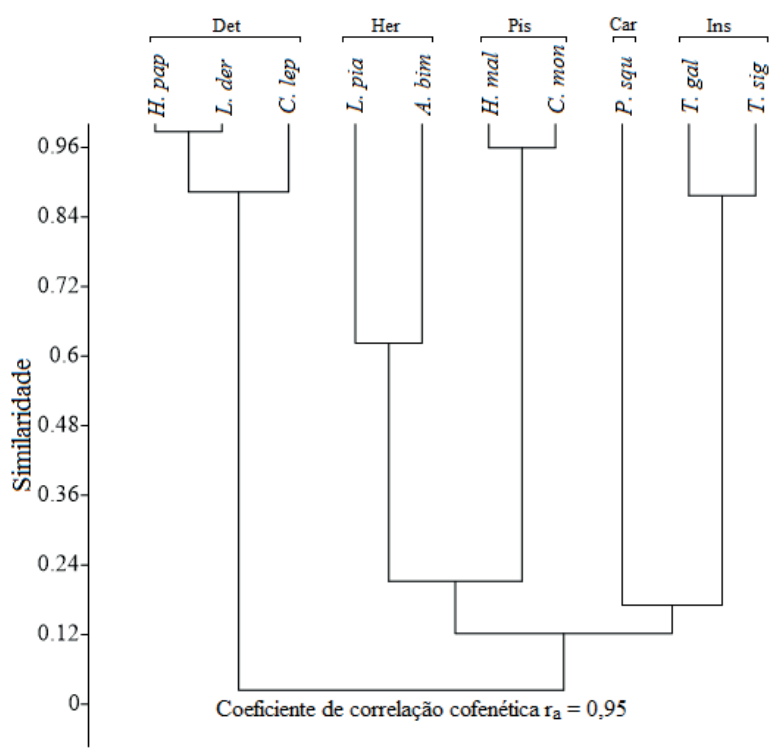

Fig. 2. Dendrograma de similaridade alimentar a partir dos valores do Índice de Importância Alimentar (IAi) das dez espécies de peixes estudadas no reservatório de Santa Cruz, Rio Grande do Norte, Brasil (Det., Detritívora/ Iliófaga; Her., Herbívora; Pis., Piscívora; Car., Carcinófaga; Ins., Insetívora). 


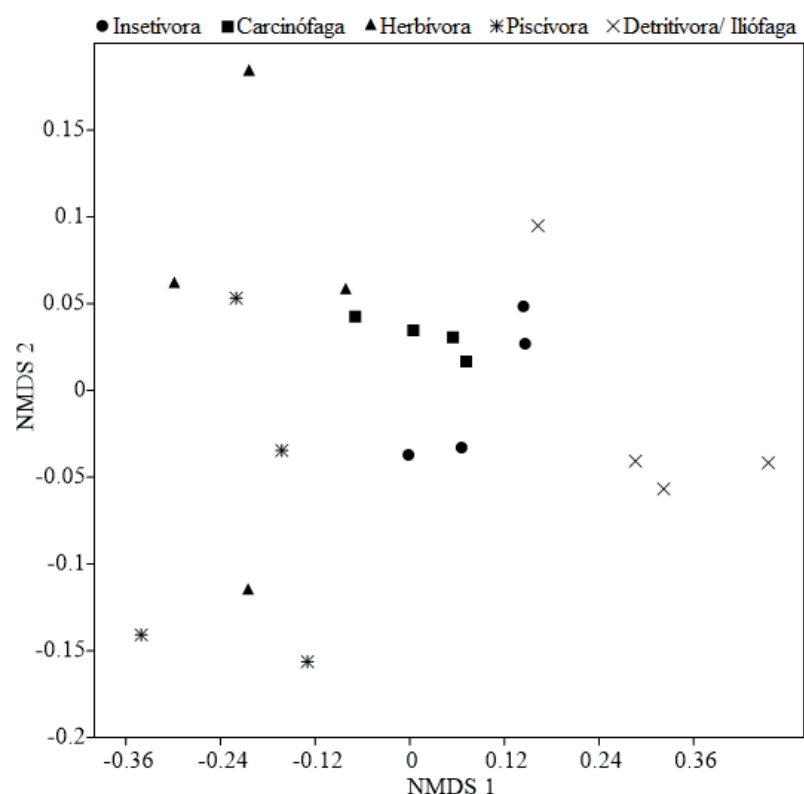

Fig. 3. Representação gráfica dos dois primeiros eixos da análise de escalonamento multidimensional não métrico (NMDS 80,4\%; Stress 0,10), para a distribuição temporal da abundância de peixes das guildas tróficas no reservatório de Santa Cruz, Rio Grande do Norte, Brasil.

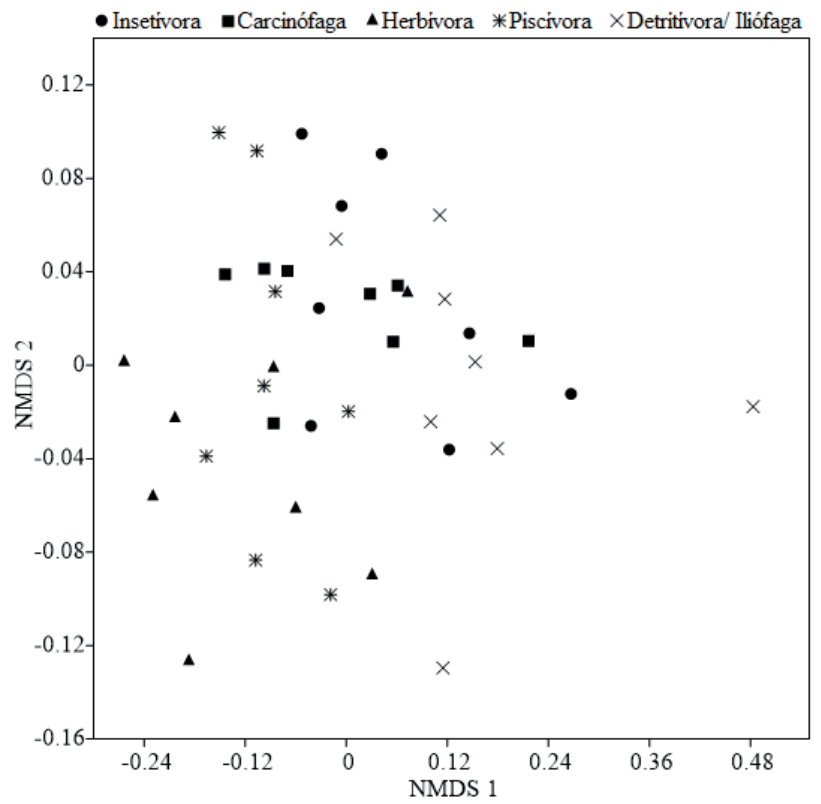

Fig. 4. Representação gráfica dos dois primeiros eixos da análise de escalonamento multidimensional não métrico (NMDS 74,6\%; Stress 0,12), para a distribuição espacial da abundância de peixes das guildas tróficas no reservatório de Santa Cruz, Rio Grande do Norte, Brasil.

Detritívora/Iliófaga. Os peixes da guilda detritívora/ iliófaga pertencentes a Loricariidae e Curimatidae representa $37 \%$ da biomassa total de peixes capturados no alto rio São Francisco, evidenciando a importância do recurso detrito/ sedimento para ictiofauna dessa bacia (Alvim \& Peret, 2004). Estudos realizados no reservatório de Juramento (MG) caracterizaram o hábito alimentar da C. lepidura como iliófaga (AlvARENGA et al., 2006). Em nosso trabalho essa espécie foi classificada como detritívora/iliófaga, pois apresentou predomínio de sedimento em relação ao detrito na sua dieta, já que a constituição do conteúdo estomacal era de partículas finas associadas a material orgânico, pouco detrito e algas unicelulares (Bacillariophyta, Chlorophyceae, Zygnemaphyceae e Cyanophyceae)

O sucesso na colonização da C. lepidura em ambientes represados pode estar associado à grande disponibilidade desses recursos alimentares (AgostinHo et al., 2007) ou pela preferência por ambientes lênticos para reprodução (SATO et al., 2003) como lagos ou barragens. Este mesmo enquadramento trófico vale para as espécies de Loricariidae (Hypostomus pusarum e Loricariichthys platymetopon), embora suas características as evidenciem mais como detritívoras. Assim, por seu hábito alimentar, os detritívoros/ iliófagos apresentam grande importância ecológica para esses ambientes, uma vez que contribuem para a ciclagem de nutrientes.

Insetívora. Representados pelas espécies que se alimentam principalmente de insetos, $T$. signatus e $T$. galeatus, que ingeriram principalmente os itens Odonata, Hymenoptera, Ephemeroptera, Orthoptera, Hemiptera e Diptera. Esse hábito alimentar apresentou certa plasticidade ao incluir restos de artrópodes, escamas de peixe, moluscos, nematódeos e microcrustáceos. Esta característica adaptativa é comum em peixes neotropicais em razão de sua maior disponibilidade dos itens no ambiente e da facilidade de ser capturado (ABELHA et al., 2001). Triportheus signatus estudada no reservatório de Salto Grande (SP) apresentou uma dieta constituída por insetos e crustáceos (HöFLING et al., 2000); no reservatório de Sobradinho (BA) e no rio Seridó (RN), a espécie consumiu insetos como item preferencial (Mendes et al., 2011; Pinto et al., 2011). Portanto, devido a esse hábito alimentar insetívoro, $T$. signatus caracterizase como boa ferramenta de amostragem da entomofauna (GALina \& HaHn, 2003).

Trachelyopterus galeatus também revelou uma dieta composta principalmente por insetos na lagoa do Jiqui (Gurgel \& CANan, 1999), no rio Piranhas-Açu, no Açude Corredor (GURGEL et al., 2002) e no reservatório de Sobradinho (BA) (PINTo et al., 2011). Os insetos representam o maior grupo existente, dos quais $10 \%$ colonizam o ambiente aquático (MCCAFFERTY, 1981), o que torna esses organismos como fonte de alimento para muitas guildas tróficas (Vidotto-Magnoni \& Carvalho, 2009). Nesse contexto, a categoria trófica atribuída para $T$. signatus e T. galeatus em Santa Cruz está em consonância com outros ambientes, podendo ser explicada pela abundância do recurso inseto.

Carcinófaga. Embora pesquisas realizadas em reservatórios de outras regiões apontem Plagioscion squamosissimus como piscívora, como demonstrado nos rios Paranapanema e Tibagi (Bennemann et al., 2006), Tietê (STEFANI \& Rocha, 2009), no reservatório de Sobradinho, BA (SANTos et al., 2014), ou como insetívora, em reservatórios do rio Tietê (SP) (RAmos et al., 2008), em nosso trabalho o camarão foi o principal item consumido pela espécie. A guilda carcinófaga também foi observada no reservatório da Estação Ecológica de Tapacurá, PE (FerReIra FilHo et al., 
2014), onde a espécie também apresentou a prevalência de Macrobrachium amazonicum na dieta.

GaVILAN-LEANDro et al. (2009) classificaram P. squamosissimus como carcinófaga na Lagoa do Piató (RN). A disponibilidade de camarão no referido ambiente foi justificada pelo grande volume de água que possibilita a dispersão dos camarões, sendo facilmente predados. De acordo com a teoria do forrageamento, os organismos são adaptados para obter alimento com o maior valor energético gastando o mínimo possível (MacArthur \& PiANKA, 1966); dessa forma, a atividade de forrageamento da pescada com o camarão no reservatório de Santa Cruz pode estar alicerçada na disponibilidade desse item, a facilidade de captura e do valor energético.

Herbívora. Leporinus piau e Astyanax bimaculatus enquadram-se nessa categoria devido ao consumo de restos vegetais. MonTENEGRo et al. (2011) ao estudarem a alimentação e a estrutura populacional de L. piau numa represa na região semiárida paraibano, classificaram a espécie como herbívora com tendência à onivoria. No entanto, alguns trabalhos atribuem às espécies de Leporinus como onívoras, os quais identificaram itens de origem vegetal e animal na dieta (Durães et al., 2001); GURGEl \& CANAN (1999) atribuiram a esta espécie a categoria de onívora com tendência à insetivoria na Lagoa do Jiqui (RN).

No rio Ceará Mirim, A. bimaculatus teve sua dieta constituída basicamente por vegetais e algas, considerados alimentos básicos. Insetos tiveram pequena participação na dieta dessa espécie, tendo sido esse alimento considerado secundário (GURGEL et al., 2005), um resultado semelhante ao aqui obtido. No entanto, na lagoa Jiqui (RN) a espécie apresentou a dieta insetívora (GURGEL et al., 2002). Já no reservatório de Corumbá (GO) teve hábito alimentar onívoro com tendência à herbivoria-insetivoria (ANDRIAN et al., 2001). Resultado semelhante foi verificado em lagoas da planície aluvial do alto rio Paraná (PA), onde a dieta foi predominantemente de insetos e vegetais (Luz et al., 2008). Isso pode ser explicado porque para muitas espécies a escolha do alimento está diretamente relacionada com a disponibilidade do recurso no ambiente, além de táticas de predação e aparato bucal (ABELHA et al., 2001).

Piscívora. Hoplias gr. malabaricus e Cichla monoculus são piscívoros, apresentando como itens principais peixe seguido por camarão. A ictiofagia também se destacou como fonte primária de alimento (CARVALHO et al., 2002; PeretTi \& ANDrian, 2004; NovakowsKi et al., 2007; CorrêA \& Piedras, 2009). Da mesma maneira, C. monoculus no reservatório em formação do rio Tocantins (NovAes et al., 2004) e no reservatório de Lajes, Rio de Janeiro (SANTOS et al., 2001) apresentou hábito alimentar piscívoro. Quanto ao aparecimento de camarão na alimentação, atribuímos à abundância desse item no reservatório de Santa Cruz, refletindo a plasticidade que possuem quanto à capacidade de aproveitar esse recurso disponível.

O caráter oportunista tem sido registrado para peixes de diferentes hábitos alimentares, sobretudo carnívoros, detritívoros e onívoros, sendo considerada uma importante estratégia para a colonização de ambientes alterados, contribuindo para o sucesso na ocupação de reservatórios (Agostinho et al., 2007). Dessa forma, não se pode afirmar que se trata de espécies especialistas, uma vez que em $H$. malabaricus já foi observada mudança em sua dieta piscívora para invertebrados devido à presença de outras espécies piscívoras, assim evidenciando propensão ao oportunismo (Pompeu \& Godinho, 2001). Dessa forma, os demais itens encontrados e identificados nos estômagos, camarão e insetos (Odonata), considerados acessórios nesse cenário, podem passar a ser preferenciais em detrimento de alterações nesse ecossistema.

Variação temporal e espacial. Observou-se que espécies detritívora/iliófaga, insetívora e carcinófaga estão mais abundantes tanto nos meses de coletas quanto nos pontos amostrados. O hábito alimentar detritívora/iliófaga apresentado por Curimatidae, Prochilodontidae e Loricariidae é favorecido por ambientes represados, sendo que a estrutura trófica das espécies está relacionada com as características do meio, que para as barragens naturalmente tende a possuir grandes quantidades de detrito (Agostinho et al., 1999; BENNEMANN et al., 2011), facilitando a exploração de alimentos e preferência por esses ambientes para reprodução (SATO et al., 2003), o que explica a prevalência dessas guildas ao longo dos meses e pontos estudados.

Os insetos representam importante fonte energética para os peixes (Lowe-MCConNell, 1987; Goulding et al., 1988; FUGI et al., 2005), sendo que estes são recursos abundantes, pois diversas espécies de insetos não possuem restrições ambientais consideráveis (GoulART \& CALLISTO, 2003; Aburaya \& Callil, 2007; Beserra et al., 2010; XIMENEs et al., 2011), o que possibilita a utilização desse recurso pelos insetívoros ao longo dos meses e pontos amostrados. A guilda Carcinófaga, como é o caso do $P$. squamosissimus ao utilizar camarão como fonte principal de alimento, mostra a tendência desse recurso não ser abundante no ambiente. Desta forma, a elevada abundância dessas guildas é explicada pelo caráter oportunista das espécies, mostrando que os recursos detrito/sedimento, insetos e camarão não são limitantes ao longo dos meses e pontos estudados no reservatório estudado.

Portanto, dentre as espécies estudadas no reservatório de Santa Cruz (RN) destacam-se como mais abundantes Curimatella lepidura, Plagioscion squamosissimus, Triportheus signatus e Hypostomus pusarum. O estudo de alimentação natural de peixes permitiu agrupar as espécies em cinco categorias tróficas, tendo como as mais representativas detritívora/iliófaga, insetívora e carcinófaga, seguidas pelas onívora e piscívora, entre os meses e pontos de amostragem. Assim, pôde-se identificar a categoria trófica a que pertencem algumas espécies da assembleia de peixes do reservatório de Santa Cruz, bem como verificar quais itens mais contribuem para suas dietas e poder inferir com mais precisão a cerca das interações existentes, possibilitando no futuro entender a complexidade das relações tróficas e dar subsídios a práticas de manejo e conservação deste ambiente antropizado. 
Agradecimentos. Ao Conselho Nacional de Desenvolvimento Científico e Tecnológico $(\mathrm{CNPq})$ e a Fundação de Apoio a Pesquisa do Estado do Rio Grande do Norte (FAPERN), pelo apoio financeiro. Ao Instituto Federal do Rio Grande do Norte (IFRN), campus de Apodi, pelo apoio logístico durante as coletas de campo.

\section{REFERÊNCIAS BIBLIOGRÁFICAS}

Abelha, M. C. F.; Agostinho, A. A. \& Goulart, E. 2001. Plasticidade trófica em peixes de água doce. Acta Scientiarum, Biological Sciences 23:425-434.

Aburaya, F. H. \& CAllil, C. T. 2007. Variação temporal de larvas de Chironomidae (Diptera) em um trecho do alto rio Paraguai, Cáceres, MT. Revista Brasileira de Zoologia 24:565-572.

Agostinho, A. A.; Gomes, L. C. \& Pelicice, F. M. 2007. Ecologia e manejo de recursos pesqueiros em reservatório do Brasil. Maringá, Nupélia EDUEM. 501p.

Agostinho, A. A.; Miranda, L. E.; Bini, L. M.; Gomes, L. C.; Thomaz, S. M. \& Suzuki, H. I. 1999. Patterns of colonization in Neotropical reservoirs, and prognoses on aging. In: Tundisi, J. G. \& STrAskrabA, $\mathrm{M}$. eds. Theoretical reservoir ecology and its applications. Leiden, International Institute of Ecology, Backhuys Publishers, p. 227-265.

Agra Filho, S. S. 2010. Conflitos ambientais e os instrumentos da política nacional de meio ambiente. Desenvolvimento e conflitos ambientais. Belo Horizonte, Editora UFMG, p. 351-359.

Almeida, A. S. S.; Cuellar, M. Z.; Costa, A. M. B. \& Amorim, R. F. 2006. Caracterização das bacias hidrográficas dos rios Apodi/Mossoró e Piranhas/Assú (RN): Mapeamento do uso do solo através das imagens do satélite CBERS 2 e análise socioeconômico. Revista FAPERN 1(4):5-9.

Alvarenga, E. R. D.; Bazzoli, N.; Santos, G. B. \& Rizzo, E. 2006. Reproductive biology and feeding of Curimatella lepidura (Eigenmann \& Eigenmann) (Pisces, Curimatidae) in Juramento reservoir, Minas Gerais, Brazil. Revista Brasileira de Zoologia 23(2):314-322.

Alvim, M. C. C. \& Peret, A. C. 2004. Food resources sustaining the fish fauna in a section of the upper São Francisco River in Três Marias, MG, Brazil. Brazilian Journal of Biology 64(2):195-202.

Andrade, H. T. A.; Nascimento, R. S. S.; Gurgel, H. C. B. \& Medeiros, J. F. 2000. Simuliidae (Diptera) integrantes da dieta de Poecilia vivipara Bloch \& Schneider, 1801 (Atheriniformes; Poeciliidae) no Rio Ceará Mirim, Estado do Rio Grande do Norte, Brasil. Entomologia y Vectores 7(1):119-122.

Andrian, F. I.; Silva, H. B. R. \& Peretti, D. 2001. Dieta de Astyanax bimaculatus (Linnaeus, 1758) (Characiformes, Characidae), da área de influência do reservatório de Corumbá, Estado de Goiás, Brasil. Acta Scientiarum, Biological Sciences 23:435-440.

Bastos, R. F.; Miranda, S. F. \& Garcia, A. M. 2013. Diet and feeding strategy of Characidium rachovii (Characiformes, Crenuchidae) in coastal plain streams of southern Brazil. Iheringia, Série Zoologia 103(4):335-341.

Bennemann, S. T.; Capra, L. G.; Galves, W. \& Shibatta, O. A. 2006. Dinâmica trófica de Plagioscion squamosissimus (Perciformes, Sciaenidae) em trechos de influência da represa Capivara (rios Paranapanema e Tibagi). Iheringia, Série Zoologia 96(1):115-119.

Bennemann, S. T.; Galves, W. \& CAPRA, L. G. 2011. Recursos alimentares utilizados pelos peixes e estrutura trófica de quatro trechos no reservatório Capivara (Rio Paranapanema). Biota Neotropica 11(1):1-9.

Beserra, E. B.; Fernandes, C. R.; Sousa, J. D.; Freitas, E. D. \& Santos, K. D. 2010. Efeito da qualidade da água no ciclo de vida e na atração para oviposição de Aedes aegypti (L.) (Diptera: Culicidae). Neotropical Entomology 39(6):1016-1023.

Bicudo, C. E. M. \& Menezes, M. 2006. Gênero de Algas de águas Continentais do Brasil. Chave para identificação e descrição. 2ed. São Carlos, Rima. 473p.

Canan, B.; Castro, H.; Gurgel, B.; Soares Nascimento, R. S.; Almeida, S.; Vieira Borges, G. \& Barbieri, G. 1997. Avaliação da comunidade de sete espécies de peixes da lagoa Boa Cicca, Nísia Floresta-RN. Revista Ceres 44:604-616.

Carvalho, L. N.; Fernandes, C. H. V. \& Moreira, V. S. S. 2002. Alimentação de Hoplias malabaricus (Bloch, 1794) (Osteichthyes, Erythrinidae) no rio Vermelho, Pantanal Sul Mato-Grossense. Revista Brasileira de Zoociências 4(2):227-236.
Corrêa, F. \& Piedras, S. R. N. 2009. Alimentação de Hoplias aff. malabaricus (Bloch, 1794) e Oligosarcus robustus Menezes, 1969 em uma lagoa sob influência estuarina, Pelotas, RS. Revista Biotemas 22(3):121-128.

Cruz, C. D. \& CARneiro, P. C. S. 2003. Modelos biométricos aplicados ao melhoramento genético. Viçosa, Universidade Federal de Viçosa. 585p.

Dias, T. S. \& Fialho, C. B. 2009. Biologia alimentar de quatro espécies simpátricas de Cheirodontinae (Characiformes, Characidae) do rio Ceará Mirim, Rio Grande do Norte. Iheringia, Série Zoologia 99(3):242-248.

Durães, R.; Pompeu, P. D. S. \& Godinho, A. A. L. 2001. Alimentação de quatro espécies de Leporinus (Characiformes, Anostomidae) durante a formação de um reservatório no sudeste do Brasil. Iheringia, Série Zoologia 90:183-191.

Espíndola, E. L. G.; Branco, M. B. C.; Fracácio, R.; Guntzel, A. M.; Moretto, E. M.; Pereira, R. H. G.; Rietzler, A. C.; Rocha, O.; Rodgher, S.; Smith, W. S. \& Tavares, K. S. 2003. Organismos Aquáticos. In: Rambaldi, D. M. \& Oliveira, D. A. S. orgs. Fragmentação de Ecossistemas: Causas, Efeitos Sobre a Biodiversidade e Recomendações de Políticas Públicas. Brasília, MMA/SBF. 510p.

Fernandes, G. W. \& Barbosa, N. P. 2012. Bombas-relógio que ameaçam a natureza. Journal of Sustainable Development 5:180-191.

Ferreira Filho, V. P.; Guerra, T. P.; Lima, M. C. S.; Teixeira, D. F.; Costa, R. R.; Araújo, I. M. S.; El-Deir, A. C. A. \& De Moura, G. J. 2014. Ecomorphological patterns with diet of Plagioscion squamosissimus (Perciformes, Scianidae) in permanent reservoir in northeastern Brazil. Iheringia, Série Zoologia 104(2):134-142.

Fugi, R.; Hahn, N. S.; Loureiro-Crippa, V. E. \& Novakowski, G. C. 2005. Estrutura trófica da ictiofauna em reservatórios. Biocenoses em reservatórios: padrões espaciais e temporais. RiMa, São Carlos, p. 185-195.

Galina, A. B. \& Hahn, N. S. 2003. Comparação da dieta de duas espécies de Triportheus (Characidae, Triportheinae), em trechos do reservatório de Manso e lagoas do rio Cuiabá, Estado do Mato Grosso. Acta Scientiarum, Biological Sciences 25(2):345-352.

Gavilan-Leandro, S. A. C.; Peretti, D.; Júnior, J. E. M. P.; Fernandes, M. A. \& JúNIOR, A. M. G. 2009. Espectro alimentar e variação sazonal da dieta de Plagioscion squamosissimus (Heckel, 1840) (Osteichthyes, Sciaenidae) na lagoa do Piató, Assu, Estado do Rio Grande do Norte, Brasil. Acta Scientiarum, Biological Sciences 31(3):285-292.

Gerking, S. D. 1994. Feeding ecology of fish. San Diego, Academic Press. 426p.

Goulart, M. \& Callisto, M. 2003. Bioindicadores de qualidade de água como ferramenta em estudos de impacto ambiental. Revista da FAPAM 2(1):156-164.

Goulding, M.; Carvalho, M. L. \& Ferreira, E. G. 1988. Rio Negro, rich life in poor water: Amazonian diversity and food chain ecology as seen through fish communities. The Hague, SPB Academic. 200p.

Gurgel, H. C. B. \& Canan, B. 1999. Feeding of six fish species in Jiqui Lagoon, eastern coast of Rio Grande do Norte, Brazil. Acta Scientiarum, Biological Sciences 21(2):243-246.

Gurgel, H. C. B.; Almeida, R. G. \& Barbieri, G. 1994. Análise qualitativa da alimentação e o coeficiente intestinal de Metynnis cf. rooseveltoi Eigenmann 1915 (Characide, Myleinae), da lagoa Redonda, Nísia Floresta, Rio Grande do Norte, Brasil. Revista Brasileira de Zoologia 11(2):331-339.

Gurgel, H. C. B.; Almeida, R. G.; Barbieri, G. \& Vieira, L. J. S. 1998. Dieta de Crenicichla lepidota Heckel, 1840 (Perciformes, Cichlidae) da lagoa Redonda, Nísia floresta/RN. Acta Scientiarum, Biological Sciences 20(2):191-194.

Gurgel, H. C. B.; Lucas, F. D. \& Souza, L. L. G. 2002. Dieta de sete espécies de peixes do semi-árido do Rio Grande do Norte, Brasil. Revista de Ictiologia 10(1/2):7-16.

Gurgel, H. D. C. B.; Silva, N. B.; Lucas, F. D. \& Souza, L. D. L. G. 2005. Alimentação da comunidade de peixes de um trecho do rio Ceará Mirim, em Umari, Taipu, Estado do Rio Grande do Norte, Brasil. Acta Scientiarum, Animal Sciences 27(2):229-233.

HAHN, N. S. \& FugI, R. 2009. Fish feeding in Brazilian reservoirs: alterations and consequences in the early stages of colonization. Oecologia Australis 11(4):469-480. 
Hahn, N. S.; Loureiro, V. E. \& Delariva, R. L. 1999. Atividade alimentar da corvina Plagioscion squamosissimus (Heckel, 1840) (Perciformes, Sciaenidae) no rio Paraná. Acta Scientiarum, Biological Sciences 21:309-314.

Hammer, Ø.; Harper, D. A. T. \& Ryan, P. D. 2001. PAST: Paleontological Statistics Software Package for education and data analysis. Palaeontologia Electronica 4.

Hellawell, J. M. \& ABel, R.1971. A rapid volumetric method for the analysis of the food of fishes. Journal of Fish Biology 3:19-37.

Higuti, J. \& Franco, G. M. S. 2001. Identificação de invertebrados para análises de conteúdo estomacais de peixes. Maringá, Nupélia EDUEM. 110p.

HöFling, J. C.; Ferreira, L. I.; Ribeiro-Neto, F. D.; Bertolim, R. B. \& Berlluzzo, A. B. 2000. Distribuição, reprodução e alimentação de Triportheus signatus (Triportheus angulatus) no reservatório de Salto Grande, Bacia do rio Piracicaba, SP, Brasil. Bioikos 14(1):16-23.

KaWAKami, E. \& VAzzoler, G. 1980. Método gráfico e estimativa de índice alimentar aplicado no estudo de alimentação de peixes. Boletim do Instituto Oceanográfico 29(2):205-207.

Lowe-McConnell, R. H. 1987. Ecological studies in tropical fish communities. Cambridge, Cambridge University Press. 382p.

Luz, K. D. G.; Abujanra, F.; Agostinho, A. A. \& Gomes, L. C. 2008. Caracterização trófica da ictiofauna de três lagoas da planície aluvial do alto rio Paraná, Brasil. Acta Scientiarum, Biological Sciences 23:401-407.

MacArthur, R. H. \& Pianka E. R. 1966. An optimal use of environment. American Naturalist 100:603- 609.

Magalhães, E. M.; Almeida, R. G.; Gurgel, H. C. B. \& Barbieri, G. 1990. Contribuição ao estudo da alimentação de Serrasalmus brandtii Reinhardt, 1874 (Characidae, Serrasalmidae) do rio Piranhas-Açu, Pendências, Rio Grande do Norte. Revista Ceres 37(213):429-442.

McCAfferty, W. P. 1981. Aquatic entomology: the fishermen's and ecologist's illustrated guide to insects and their relatives. Boston, Jones and Bartlett Publishers. 448p.

Mendes, L. B.; Borges, J. A. T.; Silva, M. J.; Costa Ramos, R. T. \& MedeIRos, E. S. F. 2011. Food habits of Triportheus signatus (Teleostei, Characidae) in a Brazilian semi-arid intermittent river. Revista Brasileira de Zoociências 13:1- 3.

Merritt, R. W. \& Cummins, K. W. 1996. An introduction to the aquatic insects of North America. 3ed. Dubuque, Kendall/Hunt. 722p.

Montenegro, L. A.; Damasceno, D. N. F.; Almeida, R. G. \& Chellappa, S. 2011. Biologia alimentar do mussum, Synbranchus marmoratus (Bloch, 1795) (Osteichthyes: Synbranchidae) no açude Marechal Dutra localizado no semi-árido brasileiro. Biota Amazônia1:52-60.

Needham, J. G. \& Needham, P. R. 1982. Guia para el estudio de los seres vivos de las aguas dulces. Barcelona, Reverté S.A. 131p.

Novaes, J. L. C.; Caramaschi, E. P. \& Winemiller, K. O. 2004. Feeding of Cichla monoculus Spix, 1829 (Teleostei: Cichlidae) during and after reservoir formation in the Tocantins River, Central Brazil. Acta Limnologica Brasiliensis 16(1):41-49.

Novakowski, G. C.; Hahn, N. S. \& Fugi, R. 2007. Alimentação de peixes piscívoros antes e após a formação do reservatório de Salto Caxias, Paraná, Brasil. Biota Neotropica 7(2):149-154.

Pacheco, C. B. \& Baumann, J. C. 2006. Apodi: Um Olhar Em Sua Biodiversidade. Natal, Edição dos autores. 364p.

Peretti, D. \& Andrian, I. F. 2004. Trophic structure of fish assembleges in five permanent lagoons of the high Paraná River floodpain, Brazil. Environmental Biology of Fishes 71:95-103.

Pessoa, E. K. R.; Lima, L. T. B.; Chellappa, N. T.; Souza, A. A. \& Chellappa, S. 2013. Aspectos alimentares e reprodutivos do cascudo Hypostomus pusarum (Starks, 1913) (Osteichthyes: Loricariidae) no açude Marechal Dutra, Rio Grande do Norte, Brasil. Biota Amazônia 3(3):45-53

Pinto, G. A.; Rocha, A. A. F. \& Lacerda, N. C. 2011. Variação sazonal na dieta de Triportheus guentheri (Garman, 1890) (Actinopterygii: Characidae), no reservatório de Sobradinho, rio São Francisco, BA. Boletim do Instiuto de Pesca 37(3):295-306.
Pompeu, P. S. \& Godinho, A. L. 2001. Mudança na dieta da traíra Hoplias malabaricus (Bloch) (Erythrinidae, Characiformes) em lagoas da bacia do rio Doce devido à introdução de peixes piscívoros. Revista Brasileira de Zoologia 18(4):1219-1225.

Ramos, I. P.; Vidotto-Magnoni, A. P. \& Carvalho, E. D. 2008. Influence of cage fish farming on the diet of dominant fish species of a Brazilian reservoir (Tietê River, High Paraná River Basin). Acta Limnologica Brasiliensis 20(3):245-252.

Raposo, R. D. M. G. \& GurGEL, H. D. C. B. 2003. Variação da alimentação natural de Serrasalmus spilopleura Kner, 1860 (Pisces, Serrasalmidae) em função do ciclo lunar e das estações do ano na lagoa de Extremoz, Rio Grande do Norte, Brasil. Acta Scientiarum, Biological Sciences 25:267-272.

Rosa, R. S.; Menezes, N. A.; Britski, H. A.; Costa, W. J. E. M. \& Groth, F. 2003. Diversidade, padrões de distribuição e conservação dos peixes da Caatinga. In: Leal, I. R.; Tabarelli, M. \& Silva, J. M. C. eds. Ecologia e Conservação da Caatinga. Recife, Editora Universitária da UFPE. 807p.

SANTANA FILHO, J. R. 2007. Projeto São Francisco: garantia hídrica como elemento dinamizador do semiárido nordestino. Inclusão Social 2(2):14-18

Santos, L. N.; Gonzalez, A. F. \& Araújo, F. G. 2001. Dieta do tucunaréamarelo Cichla monoculus (Bloch \& Schneider) (Osteichthyes, Cichlidae), no Reservatório de Lajes, Rio de Janeiro, Brasil. Revista brasileira de Zoologia 18(11):191-204

Santos, N. C. L.; Medeiros, T. N.; Rocha, A. A. F.; Dias, R. M. \& Severi, W. 2014. Uso de recursos alimentares por Plagioscion squamosissimus - piscívoro não-nativo no reservatório de Sobradinho-BA, Brasil. Boletim do Instituto de Pesca 40(3):397-408.

Sato, Y.; Fenerich-Verani, N.; Nuñer, A. P. O.; Godinho, H. P. \& VERANI, J. R.; 2003. Padrões reprodutivos de peixes da bacia do São Francisco. In: Godinho, H. P. \& Godinho, A. L. eds. Águas, peixes e pescadores do São Francisco das Minas Gerais. Belo Horizonte, PUC Minas, p. 229-274.

SEMARH. 2009. Secretaria de Recursos Hídricos e Meio Ambiente do Estado do Rio Grande do Norte. Disponível em <http://www. semarh.rn.gov.br/consulta/cBaciaDetalhe.asp?CodigoEstadual $=01>$. Acessado em 15.04.2011.

Silva, D. A.; Pessoa, E. K. R.; Costa, S. A. G. L.; Chellappa, N. T. \& Chellappa, S. 2012. Ecologia alimentar de Astyanax lacustris (Osteichthyes: Characidae) na Lagoa do Piató, Assú, Rio Grande do Norte, Brasil. Biota Amazônia 2:74-84.

Stefani, P. M. \& Rocha, O. 2009. Diet composition of Plagioscion squamosissimus (Heckel, 1840), a fish introduced into the Tietê River system. Brazil Journal of Biology 69(3):805-812.

Teixeira, J. L. A. \& Gurgel, H. C. B. 2004. Dinâmica da nutrição e alimentação natural de Steindachnerina notonota (Mirand-Ribeiro, 1937) (Pisces, Curimatidae), açude de Riacho da Cruz, Rio Grande do Norte, Brasil. Revista Brasileira de Zoociências 6(1):19-28.

Vidotto-Magnoni, A. P. \& Carvalho, E. D. 2009. Aquatic insects as the main food resource of fish the community in a Neotropical reservoir. Neotropical Ichthyology 7(4):701-708

Vitule, J. R. S. \& Prodocimo, V. 2012. Introdução de espécies não nativas e invasões biológicas. Estudos de Biologia: Ambiente e Diversidade 34(83):225-237.

Windell, J. T. \& Bowen, S. H. 1978. Methods for study of fishes diets based on analysis of stomach contents. In: BaGenal, T. ed. Methods for assessment of fish production in fresh water. Oxford, Blackwell Scientific, p. 219-226.

Ximenes, L. Q. L.; Mateus, L. A. D. F. \& Penha, J. M. F. 2011. Variação temporal e espacial na composição de guildas alimentares da ictiofauna em lagoas marginais do Rio Cuiabá, Pantanal Norte. Biota Neotropica 11(1):205-215

ZAVALA-CAMIN, L. A. 1996. Introdução aos estudos sobre alimentação natural em peixes. Maringá, Nupelia EDUEM. 129p. 